\title{
(6) OPEN ACCESS \\ Multimodal angiographic assessment of cerebral arteriovenous malformations: a pilot study
}

\author{
Raphaël Blanc, ${ }_{1}^{1}$ Aude Seiler, ${ }^{1}$ Thomas Robert, ${ }^{1}$ Humain Baharvahdat, ${ }_{1}{ }_{1}$ \\ Maxime Lafarge, ${ }^{1,3}$ Julien Savatovsky, ${ }_{1}^{4}$ Jérôme Hodel, ${ }^{5}$ Gabriele Ciccio, ${ }^{1}$ \\ Dorian Chauvet, ${ }^{6}$ Silvia Pistocchi, ${ }^{1}$ Bruno Bartolini, ${ }^{1}$ Hocine Redjem, ${ }^{1}$ Michel Piotin ${ }^{1}$
}

\begin{abstract}
- Additional material is published online only. To view please visit the journal online (http://dx.doi.org/10.1136/ neurintsurg-2014-011402).

${ }^{1}$ Department of Interventional Neuroradiology, Fondation Rothschild Hospital, Paris, France

${ }^{2}$ Neurosurgical Department, Ghaem Hospital-Mashhad University of Medical Sciences, Teheran, Iran

${ }^{3}$ Institut supérieur des biosciences, Université ParisEst- Créteil, Paris, France

${ }^{4}$ Department of Neuroradiology, Saint Joseph Hospital, Paris, France ${ }^{5}$ Department of

Neuroradiology, Fondation Rothschild Hospital, Paris, France

${ }^{6}$ Department of Neurosurgery, Hopital de la Pitié-Salpetrière, Paris, France
\end{abstract}

\section{Correspondence to} Dr R Blanc, Department of Interventional Neuroradiology, Fondation Rothschild Hospital, 25 rue Manin, Paris 75019,

France;

rblanc@fo-rothschild.fr

Received 6 August 2014 Revised 10 September 2014 Accepted 11 September 2014 Published Online First 3 October 2014

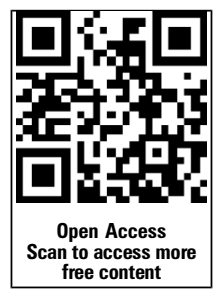

CrossMark

To cite: Blanc $R$, Seiler $A$, Robert T, et al. J

Neurolntervent Surg

2015;7:841-847.

\section{ABSTRACT}

Purpose We describe our protocol of three-dimensional (3D) Roadmap intracranial navigation and image fusion for analysis of the angioarchitecture and endovascular treatment of brain arteriovenous malformations (AVMs). Methods We performed superselective catheterization of brain AVMs feeders under 3D-Roadmap navigation. Angiograms of each catheterized artery on two registered orthogonal views were transferred to the imaging workstations, and dedicated postprocessing imaging software allowed automated multiple overlays of the arterial supply of the AVM superselective acquisitions on the global angiogram in angiographic or $3 \mathrm{D}$ views and on coregistered MRI datasets.

Results 11 untreated brain AVMs (4 with hemorrhagic presentation) were explored. The superselective acquisitions were performed under 3D-Roadmap navigation in 74 arteries, for a total of 79 targeted arteries. Imaging analysis was available at table side or postoperatively for discussion of the therapeutic strategy. No complications occurred during superselective catheterization. The accuracy of the coregistration of angiogram and MRI was submillimetric after automated mutual information coregistration, with manual reregistration by the physicians.

Conclusions Superselective angiograms acquired under 3D-Roadmap navigation can be postprocessed with multiple overlays. The fluoroscopic navigation under 3DRoadmapping and the coregistration of 3D rotational angiography, selective angiography, and 3D MR datasets appears reliable with millimeter accuracy, and could be implemented in the critical brain AVM embolization setting to allow refined analysis of AVM angioarchitecture.

\section{INTRODUCTION}

Cerebral arteriovenous malformations (AVMs) are abnormal shunts between cerebral arteries and veins without interposition of capillaries. AVMs are frequently diagnosed in young adults, aged 20-40 years, most frequently as a result of an intracranial hemorrhage or seizures. ${ }^{1}$ The annual bleeding risk is estimated as 2-33\%, with a possible 40-70\% lifetime risk. ${ }^{2}{ }^{3}$ Hemorrhage has high morbidity and mortality rates, with a $5-25 \%$ chance of death within 1 year and a $25-40 \%$ risk of permanent neurologic deficits. ${ }^{4-7}$ The treatment decision for AVMs is based on previous history of hemorrhage, lesion location, and anatomical risk factors for bleeding, such as deep brain location, deep venous drainage, initial presentation with hemorrhage, and associated aneurysms. ${ }^{2} 3$

Treatment of AVMs can be performed using open surgery, stereotactic radiosurgery, or endovascular embolization therapy. A combination of these options is sometimes preferred. ${ }^{7}$ Novel developments in imaging technologies, endovascular embolization material, as well as dedicated neuroradiology training have resulted in increased numbers of patients with AVMs that are eligible for endovascular therapy. ${ }^{8-10}$ Recent published experience notes that endovascular treatment (EVT) results in 51\% of total obliterated and $49 \%$ of incompletely obliterated AVMs with subsequent treatment. ${ }^{8}$ Depending on the series, $21-50 \%$ of AVMs are embolized prior to surgical resections in order to shorten the procedure time and prevent excessive blood loss. ${ }^{11}$

The most precise technique for description of the nidus, arterial inflow, and venous outflow of the AVM is conventional angiography, owing to its very high spatial resolution. ${ }^{12}$ Precise description of compartments in the nidus of the AVM is stressed by many authors ${ }^{13-17}$ : the presence of high flow fistulous shunts or intranidal aneurysms, compartments of the nidus core, and venous drainage of the AVM need to be recognized. During EVT of brain AVMs, superselective catheterization and angiography of the arterial feeders of the AVM are routinely performed before embolization. ${ }^{18}$ Yao et al ${ }^{19}$ have described a technique of superselective injection superimposed on the global angiogram to improve visualization of the angioarchitecture of the nidus. As threedimensional (3D) MR offers crucial anatomical landmarks, having the possibility of precisely matching those complementary modalities, it is likely to improve our analysis of AVMs prior to treatment.

We aimed to provide a superselective analysis of the arterial supply to AVMs coregistered with MR imaging prior to performing the first session of embolization. Navigation under the conditions of 3D-Roadmap and acquisitions in orthonormal registered projections were standardized, dedicated software allowed fast and precise overlay of multiple superselective angiograms on each other, and the accuracy of selective angiography and 3D MR coregistration were analyzed.

\section{METHODS}

Patients

We prospectively enrolled 11 patients harboring an untreated AVM for whom we performed a 
diagnostic 3D MRI, digitally subtracted angiography (DSA) and $3 \mathrm{D}$ rotational angiography (3D RA), superselective catheterization, and at least one session of EVT. Our local ethics committee approved the proposed protocol and written informed consent was obtained from each patient.

\section{Image acquisition}

Diagnostic 3D MRI

MR imaging was performed with a $3 \mathrm{~T}$ superconducting unit (Ingenia; Philips Healthcare, The Netherlands) using a 32 channel head coil. A slab was placed to include the whole brain from the foramen magnum to the vertex.

\section{High resolution 3D T2 TSE}

Patients were scanned prior to the procedure on a $3 \mathrm{~T}$ MR imaging system (Philips Ingenia 3T, Best, The Netherlands) using a 32 channel head coil. All examinations included a 3D TSE T2WI using the following parameters: sagittal plane covering the whole brain; 257 slices; TR/TE 2500/304, flip angle $90^{\circ}$, number of excitations 1 ; acquisition bandwidth of $637.5 \mathrm{kHz}$; FOV $240 \times 240 \mathrm{~mm}$; and voxel size $0.7 \times 0.7 \times 0.7 \mathrm{~mm}$ (interpolation of $0.5 \times 0.5 \times 0.5 \mathrm{~mm}$ ). A total of 257 sections of $0.7 \mathrm{~mm}$ thickness were obtained. The imaging time was $5 \mathrm{~min} 55 \mathrm{~s}$.

\section{Diagnostic angiographic imaging \\ Biplanar DSA}

A 6 vessel selective cerebral angiogram with acquisitions in two orthogonal incidences (anteroposterior and lateral) was performed on an Allura flat detector based biplane angiographic system (FD20/10, Philips Healthcare). We used a previously published description of arterial blood flow to AVM, ${ }^{20}$ considering the potential contribution of each major cerebral artery: middle cerebral artery, anterior cerebral artery, and posterior cerebral artery. The categories of branches supplying the AVM were defined as (1) dominant feeding artery, (2) supportive feeding artery, and (3) non-feeding artery. All acquisitions were performed on an Allura flat-detector based biplane angiographic system (FD20/10, Philips Healthcare).

\section{$3 D$ rotational angiography}

A total volume of $28 \mathrm{~mL}$ of iodine contrast material (Iomeron 300 , Bracco, Milan, Italy), at a rate of $4 \mathrm{~mL} / \mathrm{s}$, were injected. The rotation started $3 \mathrm{~s}$ after the injection for the vessels with feeding arteries to the AVM. To obtain a 3D reconstruction, 120 two-dimensional (2D) images were registered at a rate of 30 frames $/ \mathrm{s}$, from $-120^{\circ}$ to $+120^{\circ}$, at a speed of $55^{\circ} \%$. The datasets acquired into a $256^{3}$ pixel matrix were directly transferred to a 3D workstation (Xtravision, Philips Healthcare), and reconstruction of the $3 \mathrm{D}$ volume was made from $100 \%$ to $140 \%$, in order to obtain more information with a better resolution.

\section{Analysis and planning of superselective imaging}

Postprocessing of the 3D RA with advanced vessel analysis software from the Xtravision workstation (Philips Healthcare) was realized (figure 1). A precise mapping of the malformation was obtained with identification of each feeding artery, as well as arteries at the border of the AVM, by combined analysis of the vessels of interest on anteroposterior and lateral views of the angiogram and the 3D RA. A plan for superselective angiographic acquisition was established, consisting of anticipating the projection that would give the best visualization of the origin of the branches, and allow the navigation and positioning of the catheter for injection of the contrast agent.

\section{Superselective imaging}

Navigation towards branches of interest was realized under 3D-Roadmap guidance.

\section{D-Roadmap technique}

Navigation with 3D-Roadmap guidance was obtained with a machine based $3 \mathrm{D}-2 \mathrm{D}$ registration of $3 \mathrm{D}$ RA and real time $2 \mathrm{D}$ fluoroscopy data (figure 2). ${ }^{21} 22$ After 3D RA acquisition, the Xtravision workstation allowed real time overlay of these two modes of acquisition ${ }^{23}$ (conventional 2D angiogram and 3D $\mathrm{RA}$ ), regardless of any change in $\mathrm{C}$ arm position or magnification. As the acquisition of both modalities is realized by the flat detector $\mathrm{C}$ arm without any movement of the head of the patient under general anesthesia, accuracy is submillimetric. Superselective catheterization of targeted vessels was made under 3D-Roadmap by altering the $\mathrm{C}$ arm position to reach the best catheterization incidences, as scheduled in the planned analysis of the diagnostic DSA. The correspondence between 2D fluoroscopy and 3DRA corresponding projection is almost immediate (approximately $1.5 \mu \mathrm{s}$ ).

\section{Superselective angiography}

Conventional DSA runs were acquired in two orthogonal incidences (stationary anteroposterior and lateral planes) at registered magnification and field of view after manual injection of 1-2 mL of iodine contrast material (Iomeron 300, Bracco). Superselective angiograms were stored in XtravisionWorkstation and sent to the picture archiving and communication system (PACS) and a Macintosh workstation (Apple Inc, Cupertino, California, USA) with OsiriX installed (OsiriX Foundation, Geneva, Switzerland).

\section{Image postprocessing}

Each superselective angiogram was overlaid automatically and in real time with 3D RA using the Xtravision workstation (figure 3).

For the purpose of specific analysis of the AVM angioarchitecture, superselective acquisitions realized on a machine based registration in orthonormal projections could be overlaid on one another, or on the selective angiogram of other feeding arteries with the help of a software Plug-in developed for this purpose under OsiriX (see online supplemental data). The angiographic acquisitions could be overlaid with 3D MR datasets.

\section{Mutual information coregistration technique 3D MR/ angiographic fusion}

Three-dimensional MR datasets available on the PACS system of our institution (Isite, Philips Healthcare) were uploaded onto the dedicated workstation (Xtravision, Philips Healthcare) and coregistered to the perioperative 3D RA by semi-automatic registration according to the mutual information criterion (figure 4). ${ }^{24}$ This automated registration process takes less than $30 \mathrm{~s}$ per case. We validated and manually fine tuned the coregistration by applying correction in the three different planes by panning the 3D RA volume (primary volume) slice to overlay with the underlying pre-acquired MR displayed as an MIP slice (overlay volume). The time taken to realize this registration was noted.

After registration the two volumes were fused and could be handled in a $3 \mathrm{D}$ space environment by rotating, zooming, and panning with selected rendering (maximum intensity projection (MIP), multiplanar reformation (MPR)) and slice thickness options. As the $\mathrm{C}$ arm system is used to obtain the 3D RA data, 


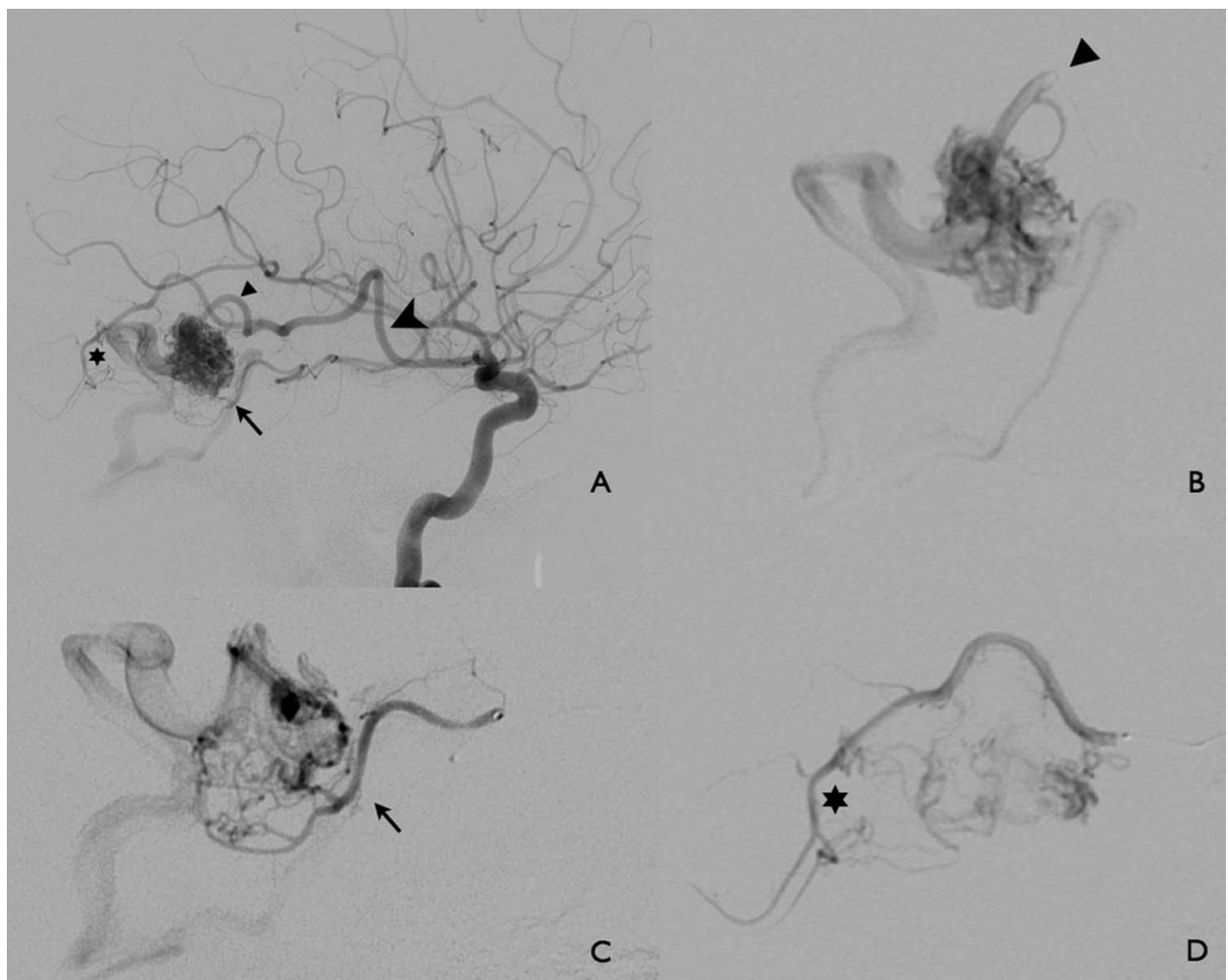

Figure 1 A patient with a previously ruptured right temporal arteriovenous malformation (AVM) (Spetzler-Martin grade 1). According to the pre-embolization analysis based on the diagnostic digitally subtracted angiography and three-dimensional rotational angiography, three branches were identified and targeted. (A) Lateral view of the selective right internal carotid artery angiogram allows analysis and planning of superselective imaging. Three feeders to the right temporal AVM are identified - a temporal dilated main trunk (large arrowhead) with a distal bifurcation giving a main feeder to the nidus (arrowhead) and a more distal posterior temporal branch with 'en passant branches' to the nidus (star); and a middle temporal artery feeder with collateral supply to the nidus (small arrow). Superselective acquisition is realized after navigating the microcatheter tip to the targeted position in each of the three feeders under three-dimensional roadmapping. (B) Lateral view of superselective injection in the main arterial feeder to the AVM, demonstrating the nidus core (arrowhead). (C) Lateral view of superselective injection in the middle temporal artery with a collateral type arterial supply to the nidus (arrow). (D) Distal posterior temporal branch with 'en passant branches' to the nidus (star).

as well as the $2 \mathrm{D}$ fluoroscopy data, the relation between their respective coordinate systems is inherently known, as long as there is no patient motion. As a consequence, the image based registration of the MR and 3D RA datasets also registered the 3D RA and C arm coordinate systems. The live fluoroscopy images and superselective angiography could thus be overlaid with the 3D RA/MR fused data.

Evaluation of the accuracy of the image based registration of 3D RA/3D MR was retrospectively and independently performed after the treatment procedure on the dedicated workstation (Xtravision, Philips Healthcare) with the aim of analyzing the maximal discrepancy between arterial feeders imaged by angiograms and arterial feeders visible on 3D MR for the image based registration technique. Using this method, we evaluated the accuracy of the registration between the vessels in the three MR planes and performed measurements at three different locations (first portion of the middle cerebral artery or posterior cerebral artery called 'proximal artery', AVM 'perinidal artery', and arterial 'cortical branch') after multiplanar reformation for HiRes3DT2 sequences.

\section{Analysis of superselective imaging}

We recorded the number of vessels that were superselectively catheterized. A classification of anatomic subgroups for these vessels, according to previously published neurosurgical ${ }^{15} \quad 25$ and neuroradiologic descriptions, ${ }^{18}$ was determined as follows: (1) terminal type arterial feeder; (2) collateral type arterial feeder; (3) 'en passant' branches; (4) normal vessels catheterized (blood flow to normal brain with no early venous drainage); and (5) arterial pedicles from border territories that corresponded to pial to pial collateral circulation.

We recorded the total number of arterial aneurysms, either proximal (visualized proximally on the circle of Willis) or more distal (observed on the superselectively catheterized branches).

We analyzed the superselective angiograms fused with the $3 \mathrm{D}$ MR datasets for correlation of vessel type with surrounding brain parenchyma.

\section{RESULTS}

Superselective imaging was performed in 11 men, aged 11-60 (mean 39) years (table 1). The principal operator who performed the superselective navigation had performed the preoperative analysis. Seventy-nine vessels were targeted for superselective catheterization. The clinical data of the patients and the results of the arterial feeder superselective mapping of the AVMs are summarized in table 1.

We were able to navigate the microcatheter in 74 out of 79 targeted positions, which gave an overall success rate of $94 \%$. Each patient's superselective angiogram could be analyzed with the dedicated software with multiple superimpositions in the anteroposterior and lateral views. These superselectively catheterized vessels were classified into five subgroups: feeders to the AVM were found for a total of 32 for terminal type arterial feeders, 16 for collateral type arterial feeders, and 4 for 'en 


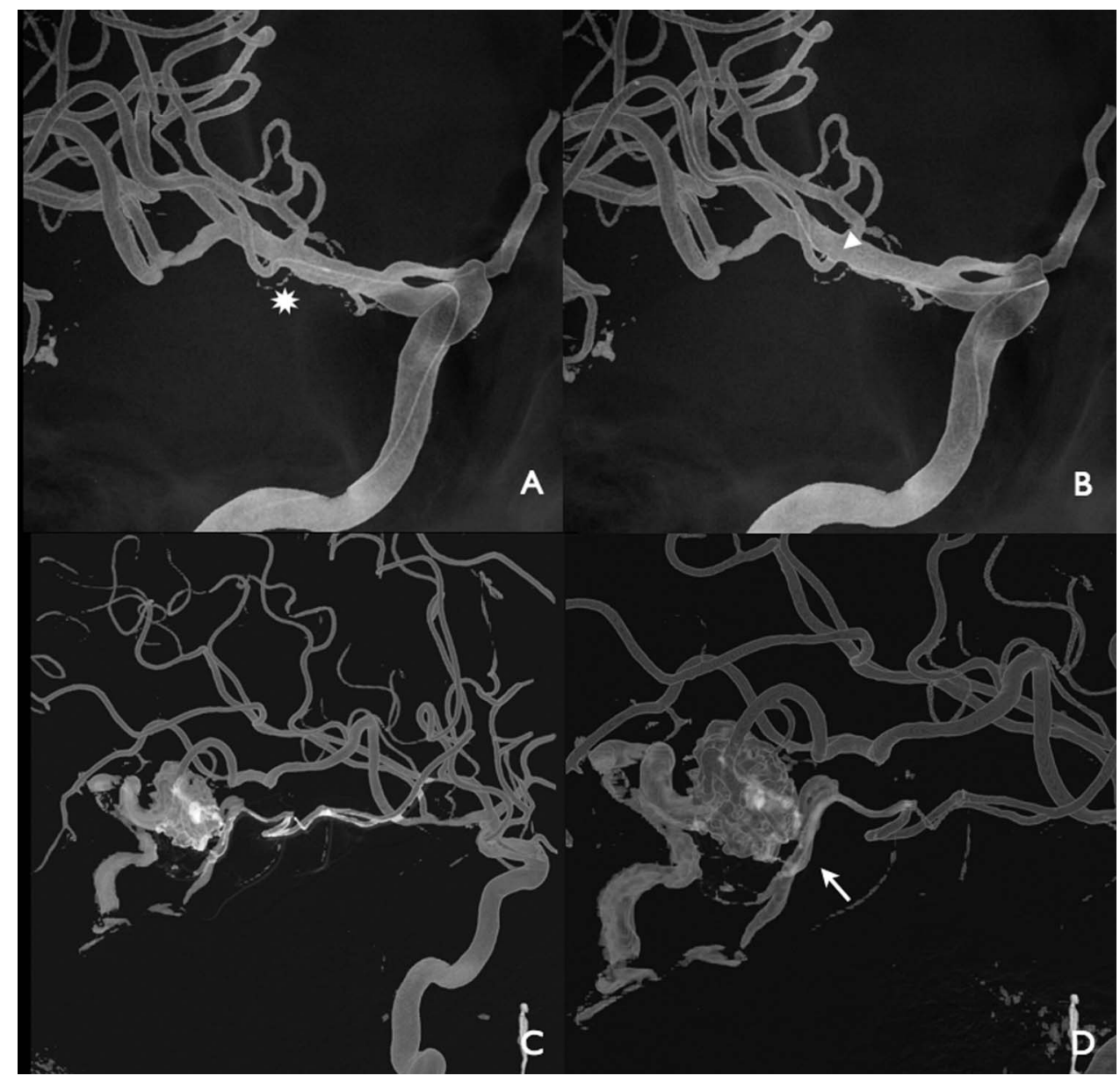

Figure 2 Three-dimensional Roadmap navigation technique of the microcatheter into the middle temporal trunk: the best scenario to visualize the origin (star) of the vessel (A) is selected by altering the position of the $\mathrm{C}$ arm flat detector, and the guide wire and microcatheter are navigated to the targeted vessels (B). Slight distortion and imperfect superimposition of the catheter on the proximal course of the artery can occur on three-dimensional images (arrowhead) but do not preclude safe catheterization. The table and patient position is not altered; manual injection through the microcatheter (C, D) during navigation and at the targeted position (arrow) are performed on two orthogonal registered incidences (lateral view shown in this case) and real time overlaid on three-dimensional rotational angiography.

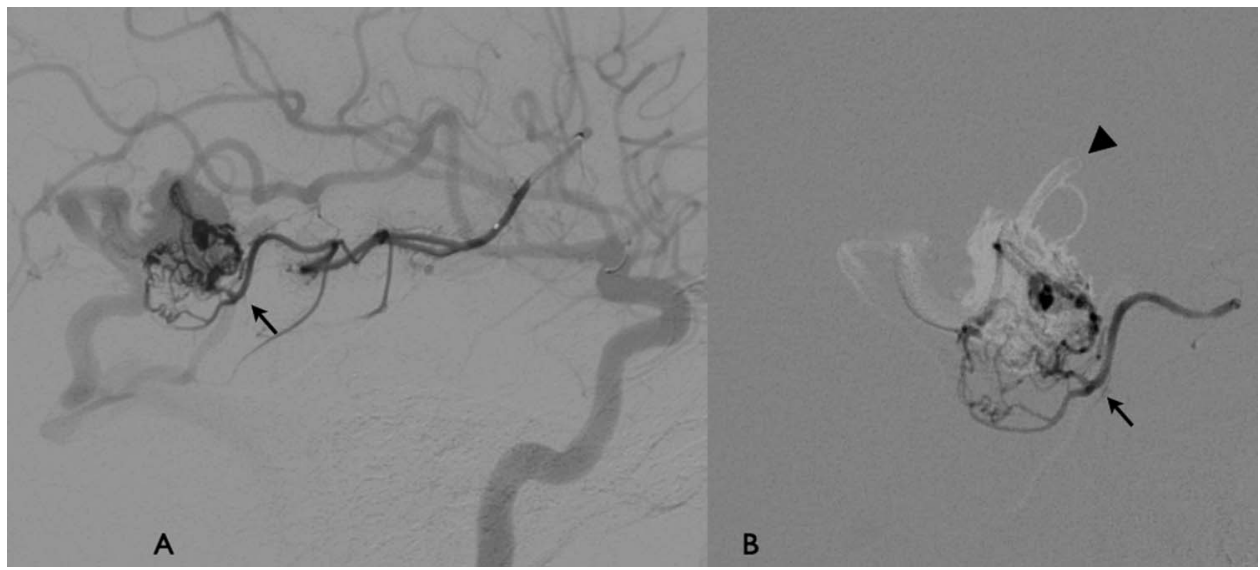

Figure 3 Acquisitions were performed in registered anteroposterior and lateral positions after those feeders were superselectively catheterized under three-dimensional Roadmap navigation. Postprocessing imaging possibilities include overlay of superselective angiogram on the selective digitally subtracted angiography (DSA), on three-dimensional rotational angiography, or on each other. (A) Postprocessing imaging: overlay of the superselective angiogram of the middle temporal branch arterial feeders (arrow) on the selective DSA (lateral view). (B) Overlay of the superselective angiogram of the middle temporal branch arterial feeders (arrow) on the superselective angiogram of the main arterial feeder to the nidus (arrowhead). 


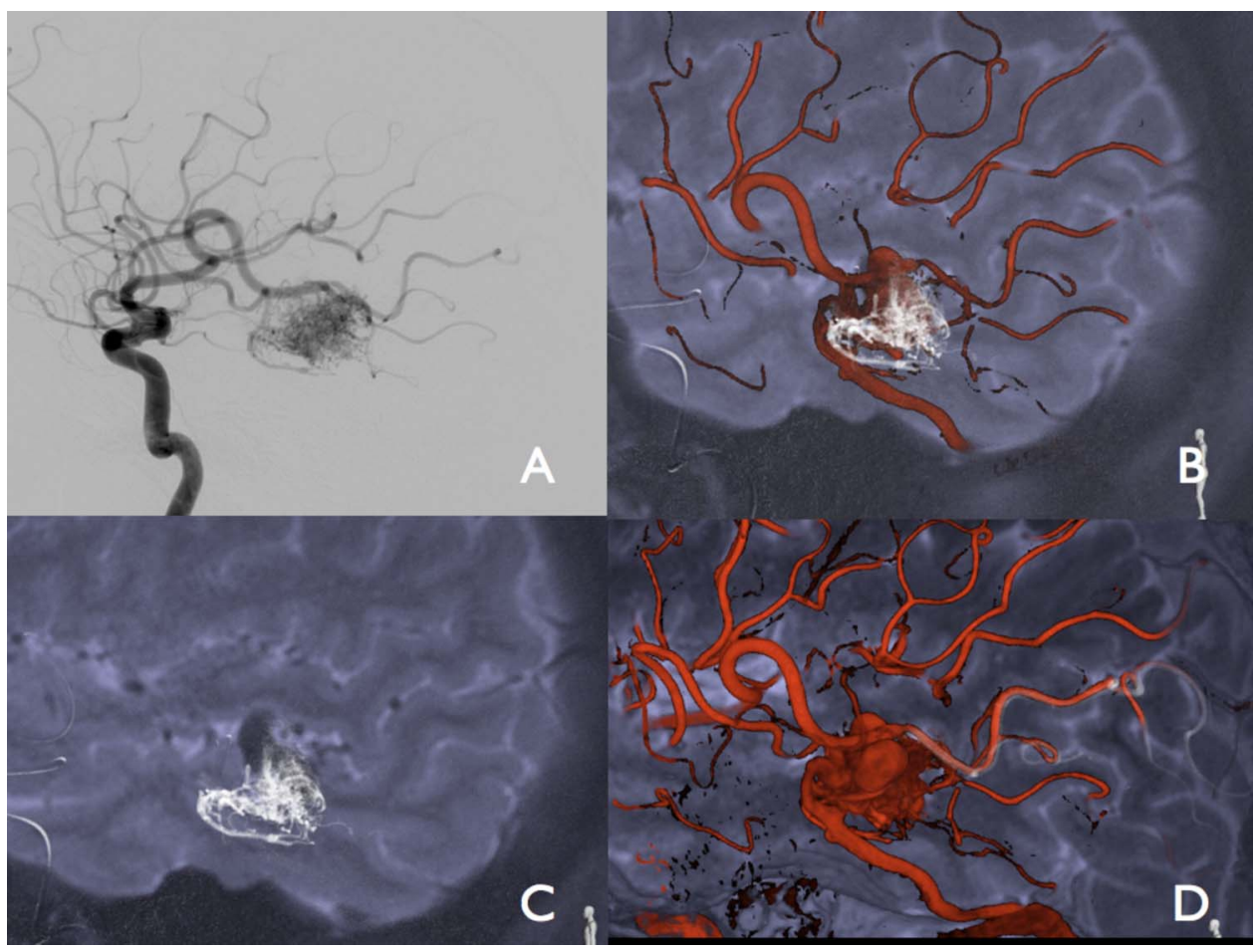

Figure 4 Patient No 4 with an unruptured left temporal arteriovenous malformation. Results of the overlay of superselective acquisition on HiresT2MRI: three-dimensional rotational angiography (3D RA) and MRI are registered according to an image based mutual information technique. As 3D RA and superselective digitally subtracted angiography are coregistered with a machine based technique with submillimetric accuracy, the image fusion of the superselective angiogram with MRI is possible, as illustrated in these images. (A) Lateral view of the left internal carotid artery. (B) Superposition of 3D RA/superselective angiogram and MR (volume rendering mode). (C) Superposition of superselective angiogram and MR, and (D) superposition of 3D RA/superselective angiogram and MR (volume rendering mode), demonstrating en passant branches with supply to the angular cortex.

passant' branches. A total of 15 normal vessels with blood flow only to normal brain with no early venous drainage were catheterized. Seven vessels corresponding to pial to pial collateral circulation were opacified.

Two proximal aneurysms were visualized on the global injections, and there were six distal aneurysms on the superselectively catheterized branches.

The mean time to perform the superselective catheterization corresponded to $30 \%$ of the total procedure time. No complications were encountered during superselective navigation or acquisition.

\section{Coregistration of 3D MR/3D RA/angiogram}

Registration with the perioperative 3D RA reconstruction took less than $1 \mathrm{~min}$, due to the efficient calculation of the mutual information criterion by employing the processing power of the graphics hardware. After the software based registration technique, a second registration process was performed by the

Table 1 Patients and summary of arteriovenous malformations

\begin{tabular}{|c|c|c|c|c|c|c|c|c|c|c|c|}
\hline $\begin{array}{l}\text { Case } \\
\text { No }\end{array}$ & $\begin{array}{l}\text { AVM } \\
\text { localization }\end{array}$ & $\begin{array}{l}\text { SM } \\
\text { grade }\end{array}$ & Hemorrhage & $\begin{array}{l}\text { Reached/ } \\
\text { targeted } \\
\text { positions }\end{array}$ & Terminal & Collateral & $\begin{array}{l}\text { En } \\
\text { passant }\end{array}$ & $\begin{array}{l}\text { Pial to } \\
\text { pial }\end{array}$ & Normal & $\begin{array}{l}\text { Proximal } \\
\text { aneurysms }\end{array}$ & $\begin{array}{l}\text { Distal } \\
\text { aneurysms }\end{array}$ \\
\hline 1 & Central & 3 & No & $9 / 9$ & 2 & 3 & 0 & 1 & 3 & 0 & 0 \\
\hline 2 & Occipital & 5 & No & $16 / 17$ & 13 & 0 & 1 & 0 & 2 & 0 & 1 \\
\hline 3 & Temporal & 1 & Yes & $4 / 4$ & 2 & 1 & 1 & 0 & 0 & 0 & 0 \\
\hline 4 & Temporal & 2 & No & $4 / 4$ & 1 & 1 & 1 & 0 & 1 & 0 & 0 \\
\hline 5 & Parietal posterior & 3 & No & $7 / 8$ & 3 & 1 & 0 & 1 & 2 & 0 & 0 \\
\hline 6 & Basi-frontal & 1 & Yes & $6 / 7$ & 3 & 2 & 0 & 0 & 1 & 1 & 0 \\
\hline 7 & Temporo-polar & 1 & No & $4 / 4$ & 1 & 2 & 0 & 0 & 1 & 0 & 0 \\
\hline 8 & Basi-frontal & 1 & Yes & $3 / 3$ & 1 & 1 & 0 & 0 & 1 & 0 & 0 \\
\hline 9 & Parietal posterior & 3 & No & $6 / 7$ & 1 & 1 & 0 & 2 & 2 & 0 & 3 \\
\hline 10 & Temporo-occipital & 5 & No & $9 / 10$ & 2 & 2 & 1 & 3 & 1 & 1 & 2 \\
\hline 11 & Temporal & 2 & Yes & $6 / 6$ & 3 & 2 & 0 & 0 & 1 & 0 & 0 \\
\hline Total & & 4 & Yes & $74 / 79$ & 32 & 16 & 4 & 7 & 15 & 2 & 6 \\
\hline
\end{tabular}

Type of arterial feeders targeted and superselectively catheterized, and presence of arterial aneurysms.

AVM, arteriovenous malformations; SM, Spetzler-Martin. 
physicians and allowed for more precise registration. This process took 1-2 min more, and thus allowed $3 \mathrm{~min}$ for the considered accurate coregistration. After automatic registration, measures ranged from 0.34 to $2.38 \mathrm{~mm}$. The mean discrepancies for 'proximal artery', 'perinidal artery', and 'cortical branch' were, respectively, $1.05( \pm$ SD 0.29$), 1.29 \pm 0.35$, and $1.22 \pm 0.24 \mathrm{~mm}$. There was no difference between the means in the three locations when compared with one another $(p>0.05)$. In fact, the maximum registration error when automatically aligning anatomic landmarks was less than $2 \mathrm{~mm}$; this error became submillimetric for HiresT2 after manual re-registration by the physicians.

\section{DISCUSSION}

Superselective catheterization is the requisite step for performing EVT by intranidal injection of embolic material. This technique and the material used are highly dependent on careful selection of the pedicles to embolize, with consideration of the potential length of reflux. Injections through the microcatheter after superselective catheterization helps to evaluate the flow dynamics of the lesion and to confirm the ideal wedge position of the microcatheter prior to performing the embolization. ${ }^{18}$

Superselective imaging can help determine the type of feeding arteries supplying the nidus core separately which can thus be classified into anatomic subgroups, according to previously published neurosurgical ${ }^{15} 25$ and neuroradiologic descriptions, ${ }^{18}$ as follows: (1) terminal type arterial feeder; (2) collateral type arterial feeder; (3) 'en passant' branches; (4) normal vessels (blood flow to normal brain with no early venous drainage); and (5) arterial pedicles from border territories corresponding to pial to pial collateral circulation.

The interest in superselective catheterization for the description of AVM compartments, or to be overlaid with more global angiography, is well known. ${ }^{13}{ }^{19}$ Many authors, including Yasargil, Lo, Yamada, and Kakizawa, ${ }^{13-17}$ have given descriptions of compartments in the nidus of the AVM. Yao et al ${ }^{19}$ have described the advantages of a technique of superselective injection superimposed with the guiding catheter injection simultaneously in the same run to improve visualization of the angioarchitecture of the nidus.

With our protocol, all superselective acquisitions are realized in orthonormal projections, allowing advanced image postprocessing on machine based or image based registration techniques to overlay with other imaging modalities. The projection used to select the origin of a feeding artery is different from the one used for navigation toward the nidus as well as the working projection to perform the injection of embolic material. The 3D-Roadmap technique allows you to switch quickly in between these various scenarios ${ }^{22}$ without patient or table movements. The processed 3D RA images are static, ignoring critical information of the angiogram, especially superimposition of the veins obscuring nidus details. The ability to overlay dynamic datasets from superselective angiography on 3D RA images is thus interesting.

The accuracy and speed of realizing the superimposition of each superselective angiogram by dedicated software greatly benefits from acquisitions in orthonormal projections. We are able, per procedure, to analyze separately or in combination each type of feeding artery supplying the AVM, as well as the arterial supply to the normal brain, which could help define a treatment strategy with regard to embolization sessions. Injection of the liquid embolic agent can be performed in a live fashion under the condition of image fusion of MR and 3D RA. The opacity of the overlay can be modified to increase the visibility of the embolic agent, and alteration of the anterior plane position can provide various incidences to refine the understanding of its deposition inside the vascular bed and especially venous outflow.

As all superselective angiograms are stored on our PACS, we are able to retrospectively analyze these datasets for refining our understanding of the complex angioarchitecture of the AVM nidus core, ${ }^{26} 27$ and scrutinize the presence of intranidal aneurysms or analyze the compartments of the AVM.

Acquisitions under the condition of 3D-Roadmap navigation allow the use of advanced coregistration with other imaging techniques, such as 3D MRI for multimodal analysis of the AVM angioarchitecture. High resolution 3D T2 anatomical sequences enable the different cerebral gyri and sulci to be clearly identified. The possibility to link the most accurate 3D RA imaging modality of the AVM nidus with 3D imaging of the brain MR and overlay fluoroscopy navigation with those 3D $\mathrm{RA} / \mathrm{MR}$ images can help in providing safer treatment.

The use of imaging fusion technique in combination with MR angiography and 2D fluoroscopic visualization for intracranial endovascular navigation and treatment ${ }^{28}$ or for endovascular therapy of aortic abdominal aneurysms ${ }^{29}$ or uterine artery embolization, ${ }^{30}$ to reduce the use of contrast agent or $\mathrm{x}$-Ray exposure, has recently been emphasized.

The anatomical information produced by this technique could also benefit other modalities of treatment, such as radiosurgery, ${ }^{31} 32$ surgical treatment of brain AVMs with roadmapping guidance, or the use of neuronavigation systems. ${ }^{33}$ In the follow-up of treated AVMs, superselective angiographic exploration could help clarify the dysplastic vessels visualized around the treated AVM that might result in further bleeding. ${ }^{34}$

However, the technique has some limitations. During microcatheterization, because of microcatheter rigidity, distortion of the course of the artery can occur on 3D images. Nonetheless, in our experience, alteration in the shape of the artery does not preclude safe catheterization and analysis of the targeted pedicle, and dedicated software is under development to address this issue. The registration technique is not fully automated and thus could be improved for better reproducibility by developing further algorithms for registration. Landmarks used for registration were mostly osseous landmarks for the 3D RA datasets, and osseous landmarks are less on the MR technique. Manual tuning of the automated registration by the operator is one step that remains cumbersome, take time, and might introduce bias.

A further limitation inherent in the techniques is that DSAMR fusion may have some image distortions, which include the distortions inherent in 3D RA and MR images, such as pin cushion distortion and $\mathrm{S}$ distortion, and those associated with image fusion. ${ }^{35}$

\section{CONCLUSION}

We have developed a protocol to describe the various types of arterial supply to the AVM. Under 3D navigation, nearly all of the direct arterial feeders to the AVM could be superselectively catheterized, and the angiograms realized in orthonormal projections allowed accurate and fast image overlays for refined analysis of the nidal angioarchitecture. We were able to superimpose the superselective angiograms of AVM nidus compartments to the precise anatomical landmarks with regards to sulci and gyri from 3D MR, providing useful information for a therapeutic approach.

Contributors All authors have made substantial contributions to the work and approved the final version of the work. 
Competing interests None.

Ethics approval Our local ethics committee approved the proposed protocol.

Provenance and peer review Not commissioned; externally peer reviewed.

Open Access This is an Open Access article distributed in accordance with the Creative Commons Attribution Non Commercial (CC BY-NC 4.0) license, which permits others to distribute, remix, adapt, build upon this work non-commercially, and license their derivative works on different terms, provided the original work is properly cited and the use is non-commercial. See: http://creativecommons.org/ licenses/by-nc/4.0/

\section{REFERENCES}

1 Stapf C, Mast H, Sciacca RR, et al. The New York Islands AVM Study: design, study progress, and initial results. Stroke 2003;34:e29-33.

2 Stapf C, Mast H, Sciacca RR, et al. Predictors of hemorrhage in patients with untreated brain arteriovenous malformation. Neurology 2006;66:1350-5.

3 da Costa L, Wallace MC, Ter Brugge KG, et al. The natural history and predictive features of hemorrhage from brain arteriovenous malformations. Stroke 2009;40:100-5.

4 Laakso A, Dashti R, Juvela S, et al. Risk of hemorrhage in patients with untreated Spetzler-Martin grade IV and V arteriovenous malformations: a long-term follow-up study in 63 patients. Neurosurgery 2011;68:372-7; discussion 78.

5 Graf $\mathrm{CJ}$, Perret GE, Torner JC. Bleeding from cerebral arteriovenous malformations as part of their natural history. J Neurosurg 1983;58:331-7.

6 Ondra SL, Troupp H, George ED, et al. The natural history of symptomatic arteriovenous malformations of the brain: a 24-year follow-up assessment. J Neurosurg 1990;73:387-91.

7 Al-Shahi R, Warlow C. A systematic review of the frequency and prognosis of arteriovenous malformations of the brain in adults. Brain 2001;124(Pt 10):1900-26.

8 Saatci I, Geyik S, Yavuz K, et al. Endovascular treatment of brain arteriovenous malformations with prolonged intranidal Onyx injection technique: long-term results in 350 consecutive patients with completed endovascular treatment course. J Neurosurg 2011;115:78-88.

9 Mounayer C, Hammami N, Piotin M, et al. Nidal embolization of brain arteriovenous malformations using Onyx in 94 patients. AJNR Am J Neuroradiol 2007;28:518-23.

10 Maimon S, Strauss I, Frolov V, et al. Brain arteriovenous malformation treatment using a combination of Onyx and a new detachable tip microcatheter, SONIC: short-term results. AJNR Am J Neuroradiol 2010;31:947-54.

11 Deruty R, Pelissou-Guyotat I, Amat D, et al. Complications after multidisciplinary treatment of cerebral arteriovenous malformations. Acta Neurochir (Wien) 1996;138:119-31.

12 Heidenreich JO, Schilling AM, Unterharnscheidt $F$, et al. Assessment of 3D-TOF-MRA at 3.0 Tesla in the characterization of the angioarchitecture of cerebral arteriovenous malformations: a preliminary study. Acta Radiol 2007;48:678-86.

13 Kakizawa $Y$, Nagashima H, Oya F, et al. Compartments in arteriovenous malformation nidi demonstrated with rotational three-dimensional digital subtraction angiography by using selective microcatheterization. Report of three cases. I Neurosurg 2002;96:770-4.

14 Lo EH, Fabrikant JI, Levy RP, et al. An experimental compartmental flow model for assessing the hemodynamic response of intracranial arteriovenous malformations to stereotactic radiosurgery. Neurosurgery 1991;28:251-9.
15 Yamada S, Brauer FS, Colohan ART, et al. Concept of arteriovenous malformation compartments and surgical management. Neurol Res 2004;26:288-300.

16 Yasargil MG. Microneurosurgery: AVM of the brain, history, embryology, pathological considerations, hemodynamics, diagnostic studies, microsurgical anatomy. New York: Thieme, 1988.

17 Yasargil MG. AVM of the brain, clinical considerations, general and special operative techniques, surgical results, nonoperated cases, cavernous and venous angiomas, neuroanesthesia. New York: Thieme, 1988.

18 Debrun GM, Aletich V, Ausman Jl, et al. Embolization of the nidus of brain arteriovenous malformations withn-butyl cyanoacrylate. Neurosurgery 1997:40:112-20.

19 Yao TL, Eskioglu E, Ayad M, et al. Improved image interpretation with combined superselective and standard angiography (double injection technique) during embolization of arteriovenous malformations. Neurosurgery 2008;62(3 Suppl 1):140-1.

20 Yamada S, Thio S, lacono RP, et al. Total blood flow to arteriovenous malformations. Neurol Res 1993;15:379-83.

21 Söderman $\mathrm{M}$, Babic D, Holmin S, et al. Brain imaging with a flat detector C-arm: Technique and clinical interest of XperCT. Neuroradiology 2008;50:863-8.

22 Söderman $M$, Babic D, Homan $R$, et al. 3D roadmap in neuroangiography: technique and clinical interest. Neuroradiology 2005;47:735-40.

23 Ruijters D, Homan R, Mielekamp P, et al. Validation of 3D multimodality roadmapping in interventional neuroradiology. Phys Med Biol 2011;56:5335-54.

24 Maes F, Collignon A, Vandermeulen D, et al. Multimodality image registration by maximization of mutual information. IEEE Trans Med Imaging 1997;16:187-98.

25 Yamada S, Brauer FS, Knierim DS. Direct approach to arteriovenous malformations in functional areas of the cerebral hemisphere. J Neurosurg 1990;72:418-25.

26 Atkinson RP, Awad IA, Batjer HH, et al. Reporting terminology for brain arteriovenous malformation clinical and radiographic features for use in clinical trials. Stroke 2001;32:1430-42.

27 lancu-Gontard D, Weill A, Guilbert F, et al. Inter- and intraobserver variability in the assessment of brain arteriovenous malformation angioarchitecture and endovascular treatment results. AJNR Am J Neuroradiol 2007;28:524-7.

28 Kocer N, Kizilkilic O, Babic D, et al. Fused magnetic resonance angiography and 2D fluoroscopic visualization for endovascular intracranial neuronavigation. J Neurosurg 2013:118:1000-2.

29 Tacher $\mathrm{V}$, Lin $\mathrm{M}$, Desgranges $\mathrm{P}$, et al. Image guidance for endovascular repair of complex aortic aneurysms: comparison of two-dimensional and three-dimensional angiography and image fusion. J Vasc Interv Radiol 2013;24:1698-706.

30 Gupta A, Grunhagen T. Live MR angiographic roadmapping for uterine artery embolization: a feasibility study. J Vasc Interv Radiol 2013;24:1690-7.

31 Colombo F, Cavedon C, Francescon P, et al. Three-dimensional angiography for radiosurgical treatment planning for arteriovenous malformations. J Neurosurg 2003:98:536-43

32 Valle RD, Zenteno M, Jaramillo J, et al. Definition of the key target volume in radiosurgical management of arteriovenous malformations: a new dynamic concept based on angiographic circulation time. J Neurosurg 2008;109(Suppl):41-50.

33 Ayad M, Ulm AJ, Yao T, et al. Real-time image guidance for open vascular neurosurgery using digital angiographic roadmapping. Neurosurgery 2007;61(3 Suppl):55-61; discussion 61-2.

34 Stapf C, Connolly ES, Schumacher HC, et al. Dysplastic vessels after surgery for brain arteriovenous malformations. Stroke 2002;33:1053-6.

35 Anxionnat $R$, Bracard S, Ducrocq $X$, et al. Intracranial aneurysms: clinical value of $3 \mathrm{D}$ digital subtraction angiography in the therapeutic decision and endovascular treatment. Radiology 2001;218(3):799-808. 